Thase, M. E. (2003) Evaluating antidepressant therapies: remission as the optimal outcome. Journal of Clinical Psychiatry, 64 (suppl. 13), 18-25.

G. Kirov Department of Psychological Medicine College of Medicine, Cardiff University, Heath Park, Cardiff CFI4 4XN, UK. E-mail: kirov@Cardiff.ac.uk

N. Khalid Whitchurch Hospital, Cardiff and Vale NHS Trust, Cardiff, UK

J. Tredget, A. Kennedy Department of Psychological Medicine, Cardiff University, UK

M. Atkins Whitchurch Hospital, Cardiff and Vale NHS Trust, Cardiff, UK

Authors' reply: We welcome the letters of Dr Kirov et al and of Dr Euba who address the important issue of clinical efficacy of electroconvulsive therapy (ECT), which may be greater when bilateral ECT is used instead of unilateral ECT. We have little doubt that this is true, but bilateral ECT is associated with more unwanted effects on cognition than unilateral ECT (National Institute for Clinical Excellence, 2003). This is the main reason why unilateral ECT is still frequently applied, certainly at the beginning of a course of treatment. Some patients experience severe and persistent memory deficits after ECT (see Donahue, 2000). In their systematic review, Rose et al (2003) found that about onethird of patients reported significant memory loss after ECT. One can question the validity of this worrisome figure on methodological grounds, as the studies reviewed by Rose $e$ al used questionnaires instead of neuropsychological assessments. Nevertheless, cognitive alterations can be very disturbing for the patient, and there remains a need to examine this controversial issue further.

In assessing the somewhat lower clinical response obtained in our study compared with others, it should be borne in mind that all our patients were treatment refractory (i.e. they had unsuccessful treatment response to at least two different types of antidepressants, each given in a sufficient dosage range for at least 4 weeks). Patients with resistance to antidepressant treatment are known to have reduced rates of response (Sackheim et al, 2000). For example, less than $30 \%$ of those with depression who had failed to respond to one adequate medication trial finally responded to low-dose or moderate-dose right unilateral ECT, in contrast to about $50 \%$ who had not received such an adequate antidepressant trial (Sackheim et al, 2000). Thus, the therapeutic effect of
ECT in our study was well within the expected range both for the group of patients studied and the type of ECT applied. It should also be noted that participants in the CORE study (Petrides et al, 2001) cited by Dr Kirov and colleagues were about 10 years older on average than patients in our study, and that ECT response rates in the CORE study were higher for older patients.

We have stated quite explicitly that our study was not designed to compare the absolute or relative effectiveness of repetitive transcranial magnetic stimulation (rTMS) or ECT. As outlined in our paper, some preliminary randomised trials suggest that rTMS might be as effective even as bilateral ECT in non-psychotic patients but, although the meta-analytic evidence for the clinical efficacy of ECT is strong, the evidence for strong efficacy of rTMS in depression is less conclusive.

Our primary intention was to highlight the continuing need to delineate the cognitive side-effects of ECT in comparison with other treatments. Weighing benefits and side-effects of a specific form of ECT treatment for a specific patient may have to take into account age, prior response to treatments, sensitivity to memory side-effects and other factors. Physicians and patients need better evidence about such sideeffects, preferably from randomised controlled trials, but also from audits such as that reported by Kirov et al, to make informed decisions on the use of ECT, particularly as other forms of treatment become available.

Donahue, A. B. (2000) Electroconvulsive therapy and memory loss: a personal journey. Journal of ECT, 16, 133-143.

National Institute for Clinical Excellence (2003) Guidance on the Use of Electroconvulsive Therapy. London: NICE (http: / /www.nice.org.uk/pdf/ 59ectfullguidance.pdf).

Petrides, G., Fink M., Hussain, M. M., et al (200I) ECTremission roles in psychotic versus nonpsychotic depressed patients: a report from CORE. Journal of ECT, I7, 244-253.

Rose, D., Fleishmann, P., Wykes, T., et al (2003) Patients' perspectives on electroconvulsive therapy: systematic review. BMJ, 326, 1363.

Sackeim, H. A., Prudic, J., Devanand, D. P., et a (2000) A prospective, randomised, double-blind comparison of bilateral and right unilateral electroconvulsive therapy at different stimulus intensities. Archives of General Psychiatry, 57, 425-434.

M. Wagner, S. Schulze-Rauschenbach,

T. Schlaepfer Department of Psychiatry, University of Bonn, Sigmund-Freud Strasse 25, D 53105 Bonn, Germany

\section{Hospital admission rates and diagnosis}

We read with interest the article by Thompson et al (2004) on changing patterns of hospital admission for adult psychiatric illness. Although they acknowledged the limitations of routinely collected admissions data, the authors reported a lower than anticipated proportion of all admissions in the schizophrenia and related psychoses categories and greater than anticipated proportions for depression and anxiety and substance misuse. A further analysis of admissions for substance misuse suggested that this did not include a large number of patients with dual diagnosis and that psychotic disorder secondary to alcohol or drug misuse accounted for around $10 \%$ of admissions for substance misuse.

On a variety of indices, Manchester has the highest level of need for mental health services in England (Glover et al, 1999). Using a similar methodology, we have analysed the 2003/4 admissions data for Manchester and found marked differences from the patterns reported by Thompson et al: $42 \%$ of admissions in Manchester were for schizophrenia and related psychoses (national average $26 \%$ ), with only $18 \%$ for depression or anxiety (national average $29.6 \%$ ) and $6.5 \%$ for substance misuse (national average $19.1 \%$ ). Further examination of the admissions for substance misuse in Manchester showed that $57 \%$ were for psychoses secondary to alcohol or drug misuse.

Our own earlier analyses of admissions in the north west of England (Harrison et al, 1995) also found marked variation according to diagnostic group and suggested that health districts with higher levels of deprivation admitted a higher proportion of patients with psychotic diagnoses and fewer patients with anxiety and depression. Similarly, the King's Fund report into London's mental health (King's Fund, 1997) argued that a high proportion of admissions for schizophrenia reflected increased need for services. This could explain some of the regional variation in admissions according to diagnostic group reported by Thompson et al and our own recent findings. Admissions for substance misuse may also be influenced by deprivation and availability of in-patient beds, with some areas only admitting patients with secondary psychoses rather than drug or alcohol dependence. 